\title{
IMPORTANCIA DEL CRECIMIENTO DEL SECTOR CONSTRUCGIÓN EN LA ECONOMÍA Y SOCIEDAD PERUANA
}

"Donde hay una empresa de éxito, alguien tomó alguna vez una decisión valiente".

\author{
Peter Drucker
}

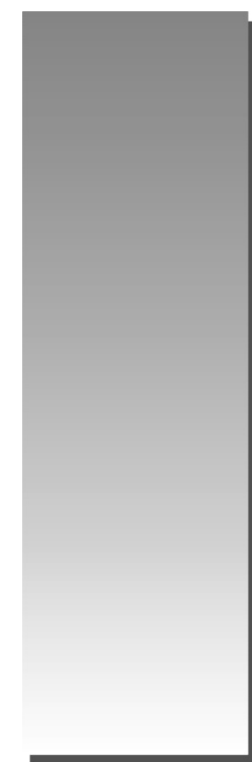

\section{Allan Vargas G.1 avargasg@unmsm.edu.pe \\ Víctor Castro M.2 vcastrom@unmsm.edu.pe}

Elena Bautista F.3 ebautistaf@unmsm.edu.pe

\section{RESUMEN}

Para el año 2011 se proyecta que el sector construcción a nivel nacional registre un crecimiento anual de $8.3 \%$, impulsado por el buen desempeño de la inversión privada, la cual crecería a una tasa estimada de $11.8 \%$, mientras que la inversión pública avanzaría ligeramente en $3.4 \%$ ante el menor presupuesto para obras de infraestructura.

Por su parte, la Macro región Norte crecería a una tasa anual de 7.6\%, como resultado de la ejecución de obras relacionadas al agro (obras de irrigación), electricidad, infraestructura vial, proyectos inmobiliarios, mineros, entre otros.

Para la Macro región Centro se estima un crecimiento anual de 8.4\% en 2011, sustentado en el desarrollo de proyectos de envergadura tales como la construcción del Muelle Norte, el tramo $\mathrm{N}^{\circ} 2$ del tren eléctrico que se tiene previsto iniciar este año, proyectos relacionados al sector eléctrico en la que destaca la hidroeléctrica Chaglla, grandes proyectos inmobiliarios, construcción de centros comerciales, infraestructura vial, entre otros.

Asimismo, se prevé que la Macro región Sur crecería 8.9\% en 2011, frente al 22.5\% del año previo, el menor dinamismo se debe a que en este año se culminan las obras faltantes de la carretera Interoceánica Sur que fueron el motor de la actividad constructora en dicha región, así también por la menor inversión pública en infraestructura. Sin embargo, la ejecución de importantes proyectos relacionados al gas natural, minería, infraestructura vial, infraestructura turística y construcción de grandes centros comerciales y conjuntos habitacionales, también influirán en el crecimiento.

Palabras claves: Dinámica del sector construcción, Perspectivas de largo plazo, Impacto económico en la economía nacional, Evaluación estratégica

1 Magister en Administración. Licenciado en Administración. Profesor Auxiliar de la Facultad de Administración de la UNMSM.

2 Estudios de Doctorado. Egresado de Maestría en Administración. Licenciado en Administración. Profesor Asociado de la Facultad de Administración de la UNMSM.

3 Estudios de Doctorado. Egresada de Maestría en Administración. Licenciada en Administración. Profesor Asociado de la Facultad de Administración de la UNMSM. 
The year 2011 projects that the national construction sector gets an annual growth of $8.3 \%$, driven by the good performance of private investment, which would grow at an estimated rate of $11.8 \%$, while public investment slightly advance at $3.4 \%$ by the lowest budget for infrastructure.

For its part, the Northern Region grows at an annual rate of 7.6\% as a result of the execution of works related to agriculture (irrigation projects), electricity, road infrastructure, real estate projects and mining, among others.

For the Central Region is expected to grow $8.4 \%$ annually at 2011, based on the development of major projects such as construction of the North Port, the second part of the electric train which is scheduled to start this year, the great projects in the hydroelectric sector like Chaglla highlighting, the large real estate projects, construction of shopping centers, road infrastructure, among others.

Also, the South Region is expected to grow $8.9 \%$ in 2011, compared with $22.5 \%$ of the previous year, the slowdown is due to the missing works of South Interoceanic Highway in this year, and the decline in public investment in infrastructure. However, the implementation of major projects related to natural gas, mining, infrastructure, tourism infrastructure and construction of large shopping centers and housing projects, also influence the growth.

Keywords: Dynamics of the construction sector, Long-term perspectives, Economic impact on the national economy, Strategic Assessment

\section{Introducción}

\section{Dinámica del Sector Construcción}

El sector construcción esta compuesto por:
A. El mercado inmobiliario
B. El mercado del cemento
C. Obras de infraestructura pública y privada

\section{A. El mercado inmobiliario}

Ver gráfico №1 El mercado inmobiliario nacional

- Demanda de viviendas mantendrá expansión por mejora en actividad económica y favorables condiciones de financiamiento.

Luego de una marcada desaceleración de las ventas de viviendas en Lima Metropolitana durante la primera mitad de 2009, estas se han venido recuperando. Las ventas orientadas a los sectores de mayores ingresos (NSE A y B) son las que vienen registrando el mayor dinamismo, explicando tres cuartas partes del total de unidades vendidas, mientras que el desempeño de las ventas a los sectores de menores ingresos es más moderado. Esta evolución se da en un mejor entorno económico, caracterizado por una fuerte expansión del PBI, un mayor dinamismo en la generación de empleo formal y una mejora de la confianza de las familias sobre la situación económica futura. Asimismo, las condiciones financieras se mantienen atractivas, con tasas de interés hipotecarias que han mostrado una tendencia a la baja desde el segundo trimestre de 2009. Se estima que estos mismos factores continuarán brindándole soporte a la demanda de este mercado en los próximos años.

En el mediano plazo, diversos factores estructurales mantendrán la dinámica del mercado. Entre ellos se encuentran: a) el actual déficit habitacional (que se estima en 300,000 viviendas en el 2009), b) las tendencias poblacionales (incremento del segmento poblacional con mayor demanda potencial), c) las posibilidades de penetración del financiamiento hipotecario (actualmente menor al 4\% del PBI), y d) los sólidos fundamentos macroeconómicos del país. A esto se añade que, según la Global Property Guide, el precio por $m_{2}$ de las viviendas en Perú se encuentra todavía algo rezagados con respecto a la región (Lima: US\$ 814/ $\mathrm{m}_{2}$, Buenos

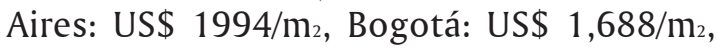
Santiago de Chile: US\$ 1,486/ $\mathrm{m}_{2}$ ).

- Oferta residencial acompañará la mayor demanda, orientándose cada vez más a los segmentos poblacionales de menores recursos

La oferta de viviendas residenciales también viene mostrando una evolución positiva en lo que va del presente año (2011). Destaca que la construcción de viviendas de precios más bajos ha empezado a dinamizarse, lo que contrasta con lo que se venía observando hasta hace un año, es decir, una oferta residencial principalmente orientada a familias de mayores recursos. 


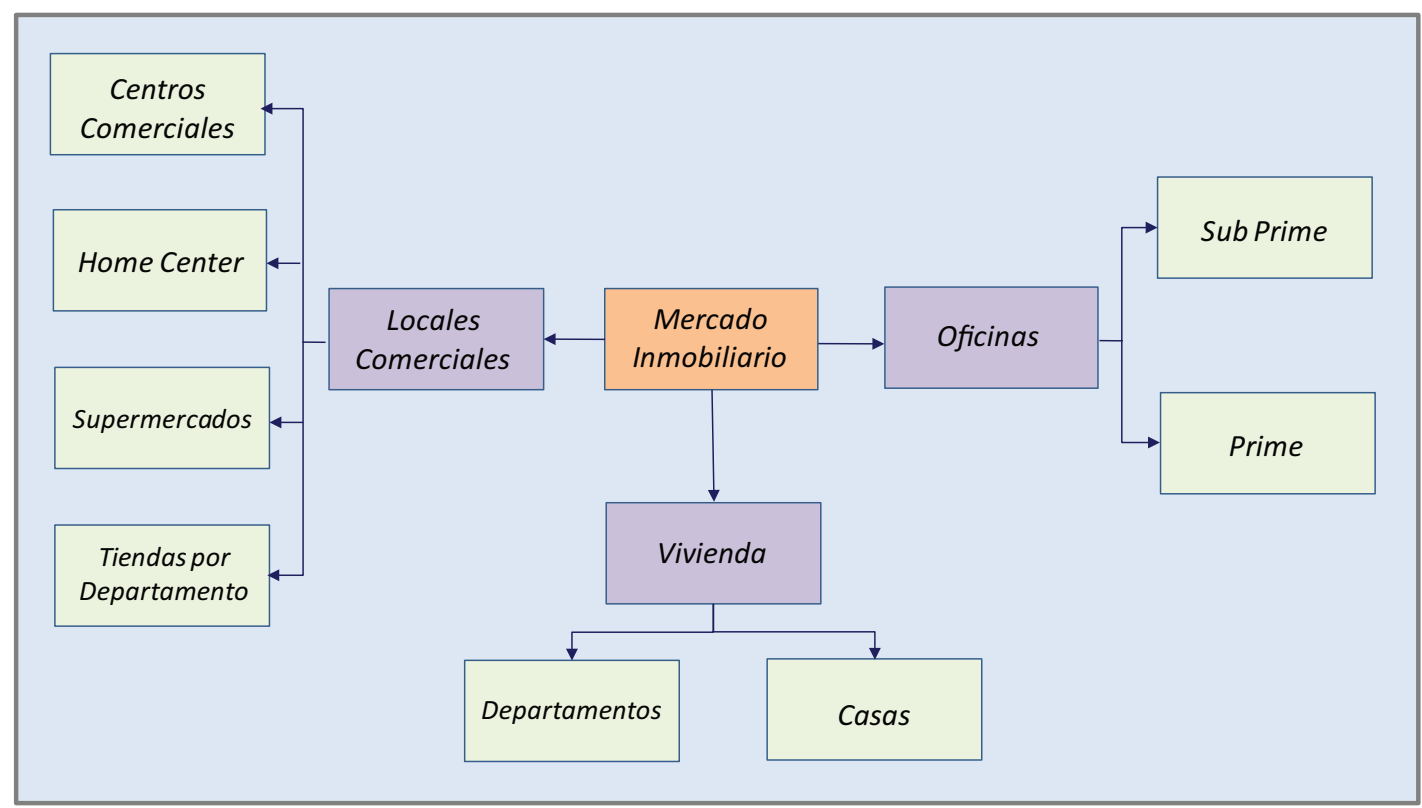

Fuente: MAXIMIXE

En los próximos años la construcción residencial continuará presentando una evolución favorable. En primer lugar, los empresarios en general tienen una visión positiva sobre la evolución futura de la economía, optimismo que se ubica en niveles máximos históricos. En segundo lugar, el costo de financiamiento para la actividad edificadora residencial se mantendrá en niveles atractivos, en un contexto de riesgo-país favorable.

\section{- Precios de los departamentos se elevan}

En el 2009, los precios de los departamentos en la ciudad de Lima se han incrementado en $10 \%$, de US\$ $741 / \mathrm{m} 2$ en el 2008 a US\$ $814 / \mathrm{m} 2$ en 2009. Este aumento, que se dio a pesar de la desaceleración de la demanda doméstica, habría estado explicado porque las viviendas fueron construidas con insumos a precios de 2008, que por entonces eran elevados. Aunque, no se dispone de información para lo que va del año, la Cámara Peruana de la Construcción (Capeco) deslizó que los precios de los terrenos aumentaron en $8 \%$ durante el primer trimestre del 2010, en un entorno de mayor demanda para construcción de viviendas en los distritos de bajos ingresos, donde los precios han llegado a aumentar más de $30 \%$.

Dados los factores mencionados, se espera que la tendencia alcista de los precios de las viviendas continúe en los próximos meses debido a: a) el incremento en los precios de los terrenos, b) el alza de los precios de los materiales de construcción y, en menor medida, c) la elevación del costo de la mano de obra. La saturación de los espacios disponibles para construcciones en distritos "exclusivos" y, en general, las expectativas de una creciente demanda continuarían presionando al alza los costos de los inmuebles. Por su parte, los costos laborales, aproximados por el salario por hora trabajada por la PEA ocupada, se han incrementado en $3 \%$ en el 2010, mientras que el aumento promedio podría ser hasta de $6 \%$ si se llega a concretar la propuesta del sindicato de trabajadores de la construcción, a negociarse con Capeco para el periodo 20102011. Finalmente, los precios de los materiales de construcción se vienen recuperando gradualmente, después de haberse contraído en $6 \%$ en promedio en 2009 , impulsados por los mayores precios de productos metálicos, vidrios, suministros eléctricos, entre otros. En este contexto, los precios de insumos podrían elevarse hasta en $9 \%$ en promedio, afectando principalmente los costos de las viviendas para los sectores $\mathrm{C}$ y D.

Considerando la estructura de costos de las empresas promotoras y constructoras, los precios de venta de las viviendas podrían elevarse hasta en 8\% en 2010. 
- Perspectivas para las inversiones en vivienda social

El Estado mantiene actualmente una activa participación en el mercado habitacional, la que tiene como fin contribuir a reducir el déficit cualitativo (estado de la vivienda) y cuantitativo en el mismo, en particular en los segmentos poblacionales de menores recursos. El apoyo estatal busca favorecer tanto a los que ofrecen como a los que demandan viviendas, y se otorga bajo la forma de facilidades financieras y de subsidios directos. Los principales programas públicos de este tipo son MiVivienda y Techo Propio. Estos canalizan recursos a través del sistema financiero. El primero de ellos brinda facilidades financieras para la adquisición de inmuebles con precios que en dólares equivalen a un rango que va de US\$ 18 mil a US\$ 60 mil, mientras que el segundo actúa en los rangos de precios más bajos de US\$ 7 mil a US\$ 18 mil.

A junio de 2010, el programa MiVivienda ha otorgado casi 2,300 créditos hipotecarios, un ritmo que es superior al de años anteriores. Este fuerte incremento estaría explicado por las campañas realizadas para promocionar los productos en moneda nacional, las mejores condiciones ofrecidas por el programa a partir de este año, así como su atractivo asociado al otorgamiento del "Bono al Buen Pagador". La meta para el presente año es otorgar 12,000 créditos hipotecarios (S/. 1,000 millones), mucho más ambiciosa que la de años anteriores. Más aun, el financiamiento otorgado es desde principios de 2008 en moneda local, lo que reduce el riesgo cambiario del prestatario. Los recursos del fisco destinados a Techo Propio en 2010 ascienden a S/. 300 millones, monto que si bien es inferior al de 2009 de S/. 450 millones (cifra excepcional por tratarse de una de las medidas del Plan de Estímulo Económico para enfrentar la crisis internacional), se encuentra por encima de los asignados en los años 2006-2008.

El presupuesto para este programa permitirá el otorgamiento de 18,000 Bonos Familiares Habitacionales (BFH) mediante 3 modalidades: a) adquisición de vivienda nueva (57\% del presupuesto), b) construcción en sitio propio (41\% del presupuesto), o c) mejoramiento de de la vivienda ( $2 \%$ del presupuesto); así como tasas de interés favorables para los créditos hipotecarios complementarios.
Al cierre del primer semestre de 2010, se ha ejecutado casi el 50\% del presupuesto asignado para estos fines. En unidades, se han otorgado $8,000 \mathrm{BFH}$, equivalente a $30 \%$ de los desembolsos totales de 2009 y muy similar a los otorgados en el año 2008 en su conjunto. Dada la elevada demanda por este producto, el Ministerio de Vivienda ha solicitado una ampliación del Presupuesto, de modo que el beneficio alcance a 30,000 familias, meta superior a la de 2009.

No obstante, si bien existe una creciente demanda para el tipo de viviendas que encajan en estos esquemas, todavía se percibe poco interés de parte de promotores y/o constructores en desarrollar proyectos de vivienda social, en especial en Techo Propio, debido, entre otros factores, a la falta de terrenos disponibles a valores que cuadren con los precios a los que podrían ser vendidas, lo que los hace poco rentables. Esta situación se complica cuando los procesos de construcción de vivienda social vía concurso público de venta de terrenos del Estado son objeto de cuestionamiento, lo que desincentiva la participación de constructores y promotores.

- Factores de demanda del mercado de oficinas: empresas más exigentes con respecto a características de edificios

En 2009, la demanda en el mercado de oficinas prime $(\mathrm{A}+\mathrm{y} \mathrm{A})$ superó ligeramente a la observada en el 2008. Esto a pesar de que la incertidumbre sobre el impacto local de la crisis financiera internacional. Con la reactivación de la inversión privada y el incremento de las expectativas empresariales, se estima que la demanda al cierre del año 2010, se ubicaría cerca de $55,000 / \mathrm{m} 2,10 \%$ mayor que en el 2009. Con respecto al perfil de demanda, las empresas estarían solicitando cada vez mejores productos con características especiales de diseño y comodidades adicionales en sus áreas de servicios.

- Factores de oferta del mercado de oficinas: mayor dinamismo en 2010

La incertidumbre sobre el impacto local de la crisis financiera internacional no afectó de manera importante la oferta en el mercado de oficinas prime en 2009, lo que estuvo en línea con el crecimiento del sector construcción. En dicho periodo ingresaron 10 edificios, lo 
que corresponde a cerca de 90,000/m2. En el primer trimestre de 2010 no se registró ingresos de nuevos edificios, pero en el segundo trimestre entrarán cinco edificios con cerca de $62,000 / \mathrm{m} 2$, el mayor incremento trimestral desde fines del 2008.

\section{B. El mercado del cemento}

En 2010, los despachos locales de cemento acumularon 8,2 millones de TM, 15,7\% más que el año anterior. El crecimiento del mercado se explica por el mayor dinamismo del sector construcción, que pasó de crecer $6,1 \%$ el 2009 a un muy probable $12,5 \%$ el 2010 . Superada la crisis internacional y la mayoría de sus secuelas posteriores, en Perú la realización de obras inmobiliarias y de infraestructura vial viene marcando el avance hacia la modernidad.

Las exportaciones de cemento crecieron en 121.3\% respecto al año 2009 (no incluye clinker) y tuvieron como principales destinos a Bolivia y Colombia. También en 2010 las importaciones aumentaron en $125.2 \%$ hasta 0,3 millones de TM, impulsado por el fuerte dinamismo del sector construcción. Cabe resaltar el sustancial crecimiento de las importaciones de clinker, aumentaron $1,803.8 \%$ respecto al año 2009 sumando 0,7 millones de TM. En contrapartida, las exportaciones de clinker prácticamente desaparecieron.

La gran demanda insatisfecha de viviendas, el gran déficit de infraestructura y la posibilidad de obtener buenos rendimientos por su inversión hace atractivo el mercado para las cementeras extranjeras. Así se ha anunciado la instalación de cuatro nuevas plantas cementeras en el país, algunas empezarían a construir este año. Estas empresas son: Planta Cemex, Cementos Portland, Cementos Otorongo y Cementos Interoceánicos.
Gráfico №2 Ranking de Empresas Cementeras

\% Respecto a los despachos totales 2010

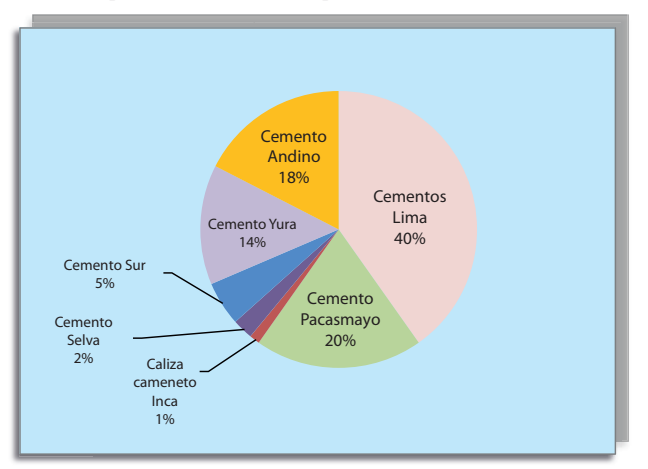

Fuente: MAXIMIXE

Obras de infraestructura pública y privada

El avance físico de obras registró una caída de $24,0 \%$ en junio de 2011 , respecto al mismo mes del año anterior. Este retroceso se debió al menor gasto en obras como: la rehabilitación y mejora de la carretera Ayacucho-Abancay; carretera CasmaYaután-Huaraz; Churín-Oyón en Lima; carretera Lunahuaná-desvío Yauyos-Chupaca; carretera Sullana-El Alamor del Eje vial $\mathrm{N}^{\circ} 2$ de interconexión vial Perú-Ecuador ; carretera Tocache-Tocache en San Martín ; Tingo María-Aguaytía- Pucallpa en Huánuco y Ucayali; rehabilitación de la carretera Tarapoto-Juanjuí en San Martín; carretera AllhuayCotahuasi Cotahuasi -La unión en Arequipa; carretera Huambutio -Paucartambo en el Cusco; carretera Yanahuanca-Cerro de Pasco; mejoramiento de la carretera Juliaca-Coata- CapachicaPusi-Taraco-empalme R3S IIPa Huata-Coata; carretera Camaná-desvío Quilca-Matarani- Ilo Tacna; mejoramiento de las avenidas Madre de Dios-La Joya y Aeropuerto-Puerto Maldonado- Madre de Dios; mejoramiento de la carretera vecinal ruta 543 Santa Lucía de Salinas-Yalagua- Lloque en Moquegua; mejoramiento de la carretera CuscoOccopata, y carretera Azángaro- SaytocochaSandia- San Juan del Oro.

Cuadro №1 Principales Proyectos de inversión Pública y Privada a Ejecutarse en el 2011

\begin{tabular}{|l|l|l|}
\hline \multicolumn{1}{|c|}{ Proyecto } & \multicolumn{1}{|c|}{ Empresa } & \multicolumn{1}{c|}{$\begin{array}{c}\text { Inversión (Millones de } \\
\text { US\$) }\end{array}$} \\
\hline Toromocho & Chinalco & 2.200 \\
\hline Antapaccay & Xstrata Copper & 1.470 \\
\hline Antamina & $\begin{array}{l}\text { Antamina (Xstrata, BHP, Teck, Mitsu- } \\
\text { bishi) }\end{array}$ & 1.300 \\
\hline $\begin{array}{l}\text { La ampliación del gasoducto } \\
\text { TGP II }\end{array}$ & TGP (Tecgas, Hunt) & 770 \\
\hline Planta Nitrato de amonio & Nitratos del Perú & 650 \\
\hline Amp. Toquepala & Southern Copper Corp. & 600 \\
\hline
\end{tabular}




\begin{tabular}{|l|l|l|}
\hline Tren Eléctrico de Lima & Odebrecht \& GyM & 500 \\
\hline Trasvase Olmos & Odebrecht & 470 \\
\hline Red Vial 4 & Cons. Español OHL Concesiones & 360 \\
\hline Autopista del Sol & Hidalgo e Hidalgo \& CASA & 360 \\
\hline La Alameda del Rímac & Miranda Constructores & 279 \\
\hline Cementos Interoceánicos & Odebrecht & 250 \\
\hline Cemex & Latinamerican Trading & 230 \\
\hline Ampliación Cementos Yura & Grupo Gloria & 220 \\
\hline $\begin{array}{l}\text { Rehabilitación Av. Néstor Gam- } \\
\text { betta }\end{array}$ & Consorcio Andrade Gutiérrez & 183 \\
\hline $\begin{array}{l}\text { Ampliación Planta Cemento } \\
\text { Andino }\end{array}$ & ARPL Tecnología Industrial S.A. & 162 \\
\hline Cementos Portland & $\begin{array}{l}\text { Bío Bío, Votarantim, Portland y World } \\
\text { Cement }\end{array}$ & 150 \\
\hline Planta de tratamiento Taboada & $\begin{array}{l}\text { ACS Servicios, Comunicaciones y Ener- } \\
\text { gía de España }\end{array}$ & 149 \\
\hline $\begin{array}{l}\text { Ampliación Planta Atocongo - } \\
\text { Cementos Lima }\end{array}$ & ARPL Tecnología Industrial S.A. & 146 \\
\hline IIRSA Centro tramo II & Concay, Incoequipos, Nexus & 100 \\
\hline Majes SIGUAS II & $\begin{array}{l}\text { AUTODEMA, Gobierno Regional de } \\
\text { Arequipa }\end{array}$ & 150 \\
\hline Ciudad Sol de Collique & Consorcio DHMONT \& CG \& MSAC & 360 \\
\hline
\end{tabular}

Fuente: MAXIMIXE

\section{MÉTODOS}

El tipo de investigación desarrollada es de carácter exploratoria ya que realiza un análisis situacional, estudia los hechos históricos y analiza posibles escenarios mediante entrevistas a profundidad a expertos en la industria de construcción, así como también fuentes secundarias como estudios de entidades especializadas como Apoyo y Maximixe, bibliografía especializada y fuentes gubernamentales como SNI, INEI, BCRP, MEF, entre otros.

\section{RESULTADOS}

- El sector construcción contribuye significativamente al crecimiento del país

El PBI del sector construcción ha tenido un crecimiento promedio anual de $10.5 \%$ durante el periodo 2005-2009. Asimismo, el año 2010 presentó una tasa de crecimiento del $12.5 \%$. Las proyecciones del BCR señalan que el crecimiento del PBI del sector construcción para el año 2011 será de 9.5\% versus el 5.5\% del PBI total. (ver gráfico $\mathrm{N}^{\circ} 3$ )

- El sector financiero cumple un papel importante en el desarrollo del sector construcción.
El Sistema Financiero Peruano viene apoyando decididamente el "boom inmobiliario y constructor" a través de líneas de financiamiento. Los créditos hipotecarios crecieron $25 \%$ en promedio en los últimos 4 años; y los créditos para la construcción lo hicieron en 33\%. Del año 2006 al año 2009, las tasas de interés en MN se mantuvieron entre $9 \%$ y $10 \%$, excepto a fines de 2008 e inicios de 2009 cuando llegaron a estar por encima de $10.5 \%$. En 2011 las tasas de interes están entre $9.8 \%$ en MN y $9.0 \%$ en ME. (ver gráficos $\mathrm{N}^{\circ} 4$ y 5 )

\section{DISCUSIÓN}

\section{Análisis Estratégico del Sector Construcción Oportunidades $^{4}$}

- El distrito de Paracas alberga inversiones que bordean los US\$ 200 millones. El presidente de la Cámara Nacional del Turismo, Carlos Canales, estimó que los proyectos de inversión se concentran en el sector hotelero. Asimismo, señaló que gracias al turismo Ica registraría un movimiento económico de US\$ 600 millones.

- JW Marriott Cusco quedará listo en el 2012 con inversión de US\$ 45 millones. Una de las 
Gráfico №3 PBI Total vs. PBI Sector Construcción

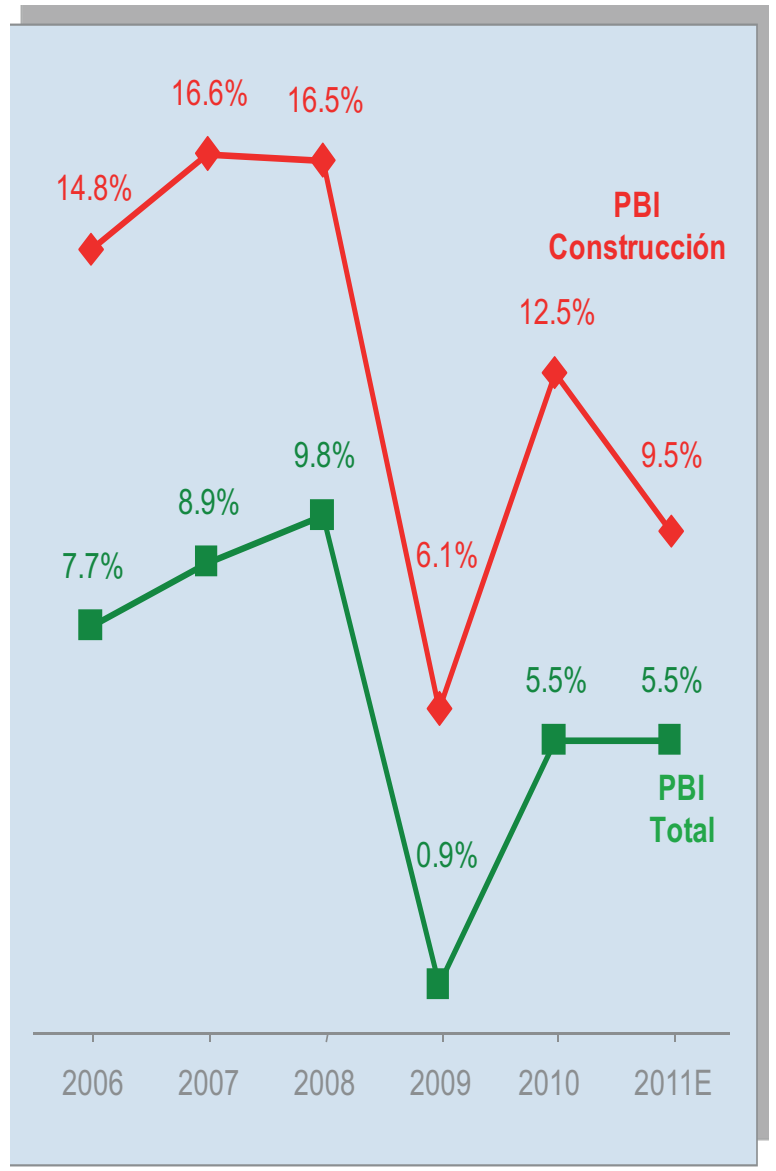

Fuente: Banco Central de Reserva del Perú

principales cadenas de hoteleras del mundo edificará un hotel de cinco estrellas y 153 habitaciones en el Cusco, el cual estará listo a más tardar el 12 de junio del 2012. Posteriormente la empresa hotelera lanzará la marca de hotel Courtyard by Marriott en San Isidro, dicha construcción finalizaría a fines de 2012.

- El Estado otorga concesiones en infraestructura que bordean los US\$ 6,813 millones. El Organismo Supervisor de la inversión en Infraestructura de Transporte de Uso Público (Ositran) anunció que estas concesiones se han realizado hasta junio de 2011. Asimismo, el organismo anunció que este año se entregaron en concesión cuatro proyectos (uno de ellos es el terminal de Yurimaguas).

- MEM autoriza inicio de proyecto minero Tantahuatay. La mina es operada por el consorcio Coimolache constituido por Buenaventura, Southern Cooper Corporation y la empresa ESPRO cuyas participaciones en el grupo son de $40.09 \%, 44.24 \%$ y $15.67 \%$, respectivamente. La producción de oro de este proyecto es de
Gráfico №4 TEA Promedio de los Créditos Hipotecarios

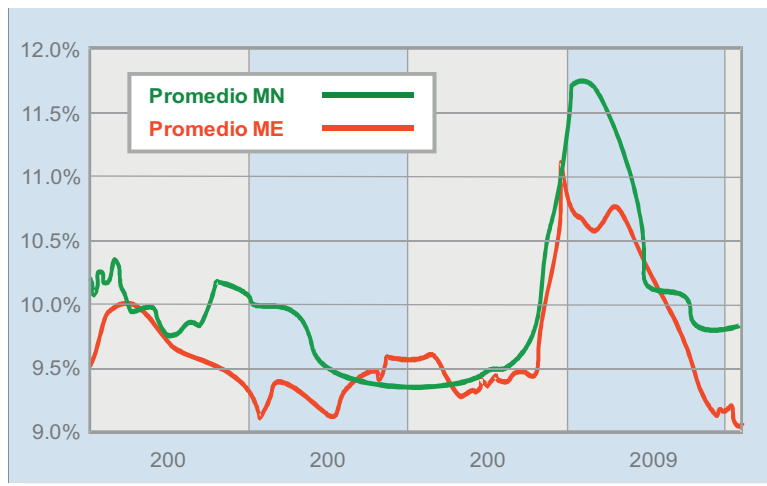

Fuente: Superintendecia de Banca y Seguros

Gráfico №5 Participación de Créditos Hipotecarios 2011

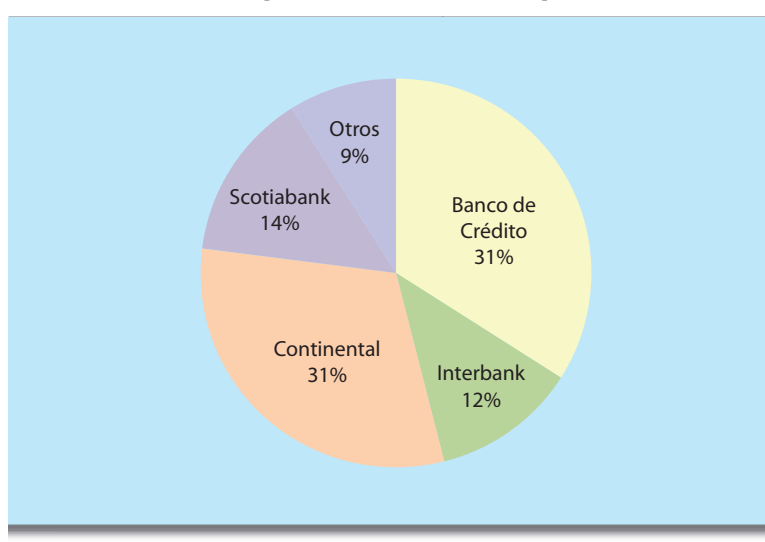

Fuente: Superintendecia de Banca y Seguros

100,000 onzas anuales y cuenta con recursos de 27.1 millones de mineral con un promedio de 13 gramos de plata y 0.89 gramos de oro por tonelada.

- El distrito de Surco Albergará un proyecto habitacional de lujo cuya inversión bordea los US\$ 37 millones. La obra será realizada por la empresa $\mathrm{C}$ y J Constructores Contratistas y consta de cinco torres de viviendas multifamiliares con 150 departamentos. El área construida que ocupará el proyecto será de $50,000 \mathrm{~m} 2$ y dispondrá de departamentos de uno y tres dormitorios con piscina, terrazas y jardines, así como áreas comunes.

- El $67 \%$ de la oferta de departamentos está enfocado en el nivel socioeconómico de estratos medios. El consumidor perteneciente al segmento socioeconómico B cuenta con una oferta disponible de 14,179 departamentos que se sumaron hasta junio de 2011 y cuyos precios no superan los S/. 200,000. 


\section{Riesgos}

- La crisis financiera internacional tuvo su origen en la fuerte expansión de los créditos hipotecarios de alto riesgo (subprime), estimulada por el exceso de liquidez en el sistema financiero norteamericano; que la política monetaria expansiva de la Reserva Federal (FED) generó con la reducción de los tipos de interés y el acceso al crédito hipotecario a grandes masas de prestatarios de dudosa capacidad financiera. Las entidades financieras dieron créditos sin mayor regulación y teniendo como colateral el propio inmueble entregado. Cuando los prestatarios entraron en moratoria, fueron ejecutadas las garantías y rápidamente se fue expandiendo el proceso como una reacción en cadena que contagió a diferentes Estados y deterioró el valor de los inmuebles. La situación se reflejó en la bolsa, donde el valor de las entidades financieras descendió rápida y progresivamente.

\section{CONCLUSIONES}

- Sector construcción continuará siendo uno de los principales motores del crecimiento económico de nuestro país aproximadamente $8 \%$ anual sostenido para los próximos tres años.

- Gran potencial de desarrollo en proyectos de infraestructura pública y privada.

- Ingreso de nuevas empresas constructoras extranjeras motivados por el crecimiento económico del país.

- Alto potencial en el mercado de construcción de viviendas en los niveles socioeconómicos B, C y D.

- Se han creado fondos de inversión inmobiliarias, existiendo en cartera fondos con capital estimado en más de $\$ 700$ MM.

- El Sistema Financiero Peruano viene apoyando decididamente el "boom inmobiliario y constructor" a través del financiamiento de créditos hipotecarios, los cuales crecieron en un $25 \%$ en promedio en los últimos 4 años; y los créditos para construcción lo hicieron en $33 \%$ al año.

\section{LITERATURA CITADA}

\section{Bibliografía de textos}

- David, Fred, La Gerencia Estratégica, cuarta edición, Fondo editorial Legis, Colombia, 1991.

- Hitt, Michael, Administración Estratégica, Thompson Learning, México 2004.

- Malhotra K. Naresh, Investigación de Mercados, México, Prentice Hall, 2004.

- Porter, Michael, Ventaja Competitiva, México, CECSA, 2004.

\section{Bibliografía de Investigaciones}

- BBVA Research, Perú - Situación Inmobiliaria, Junio 2010.

- Banco Central de Reserva del Perú - Reporte de Inflación 2011.

- Marco Macroeconómico Multianual 20112014 del MEF.

- Ipsos, Apoyo Opinión y Mercado, Setiembre 2010.

- Maximixe, Mercado inmobiliario, Marzo 2010.

- Maximixe, Mercado Cemento, Enero 2011.

- Maximixe, Riesgos sectoriales, Agosto 2011.

Bibliografía de páginas web

- http://www.mef.gob.pe

- http://www.conasev.gob.pe

- http://www.bcrp.gob.pe

- http://www.inei.gob.pe 